\title{
Zwerge im Heuhaufen
}

Die sprichwörtliche Nadel im Heuhaufen lässt sich bekannterweise nur schwer finden. Nanopartikel bzw. Nanoobjekte liegen in der Größenordnung von ca. einem Millionstel des Durchmessers einer gebräuchlichen Stecknadel (1 mm angenommen). Natürliche Nanopartikel sind beinahe ubiquitär in den Umweltmedien Wasser, Luft und Boden zu finden und werden zunehmend in künstlich hergestellter (synthetisierter) Form über eine Vielfalt an neuen Materialien und Konsumprodukten zusätzlich eingetragen. Viele dieser Trägermedien sind weitaus heterogener als der „klassische Heuhaufen“. „Nano“ ist nicht neu, natürliche Nanopartikel hat es immer schon gegeben. „Nano“ sagt auch nichts über eine spezifische Wirkung auf Mensch und Umwelt aus. „Nano“ ist prinzipiell nur die Bezeichnung einer Größenordnung, nämlich des Skalenbereiches zwischen 1 und 100 nm, daher leitet sich der Name auch vom griechischen Wort für „Zwerg“ her.

Was wir wissen, ist, dass sich Nanopartikel durch ihre große spezifische Oberfläche sowie ihr weites Oberflächen-zu-Volumen-Verhältnis auszeichnen und dadurch im Vergleich zu ihren mikroskaligen Pendants besondere „nano-spezifische“, zum Teil wesentlich reaktivere Eigenschaften aufweisen und damit eine veränderte Wirkungsweise und auch ein schwerer einschätzbares Umweltverhalten als ihre chemischen Verwandten mit sich bringen können. Zudem ist der menschliche Geist sehr erfindungsreich und die Forschungen in der Nanotechnologie sind in den letzten Jahren massiv vorangeschritten. Neue synthetisierte Nanomaterialien mit zum Teil spezifisch designten, funktionalisierten sprich deutlich von natürlichen Nanopartikeln abweichenden - Oberflächen ermöglichen es, mit sehr geringem Materialeinsatz eine ausgesprochen große Wirkung zu erzeugen, was viele Aktivitäten in unserem alltäglichen Leben erleichtert und effizienter macht. Unter anderem helfen diese innovativen Materialien und Produkte während ihrer Gebrauchsphase Energie, Betriebsmittel und Ressourcen zu sparen, könnten aber am Ende ihrer Nutzungsphase eine entsprechend zielgerichtete Verwertung und Entsorgung erschweren bzw. zusätzliche Schutzmaßnahmen für ArbeitnehmerInnen im Abfallbehandlungsbereich erfordern. Auch ist in den wenigsten Fällen ausreichend bekannt, ob und in welcher Form Nanopartikel bereits während ihrer Nutzungsphase unkontrolliert freigesetzt werden, z. B. auch über den Wasser- oder Abwasserpfad. Belastbare Dokumentationen und Forschungsergebnisse dazu stehen weitgehend aus. Von der verlässlichen Kenntnis einer potenziellen toxischen Wirkung auf den Menschen ganz zu schweigen.

Ein bisschen erinnert mich diese derzeitige Entwicklung im Nanotechnologiesektor an die wissenschaftlich wie auch politisch geführten Diskussionen betreff der synthetisch hergestellten halogenierten Kohlenwasserstoffe in den 1970er/80er-Jahren: Bereits Ende des 19. Jahrhunderts wurden die ersten halogenierten Kohlenwasserstoffe synthetisch hergestellt und fanden rasanten Absatz in unterschiedlichen Anwendungsgebieten, nicht zuletzt aufgrund ihrer willkommenen chemischen Eigenschaften, ihrer Beständigkeit, Reaktionsträgheit und - wenn überhaupt - meist nur geringen Toxizität. Zu Beginn der 1970er-Jahre wurde in wissenschaftlichen Kreisen erstmals drauf hingewiesen, dass diese Substanzen allerdings eine Ozonschicht-zerstörende Wirkung aufweisen, aber erst der Nachweis des Ozonlochs 1985 sorgte für einen umfassenderen Meinungswandel und eine breitere Wahrnehmung in der Öffentlichkeit. Die Wirkung einiger synthetisierter FCKWs als sehr potente Treibhausgase wurde ebenfalls erst in den 1980ern weitreichender bekannt. Hierbei ging es also um Wirkungen, die sich nicht in unserem unmittelbaren Umfeld, in unserer belebten Biosphäre erkennbar zeigten, aber dennoch massive Folgewirkung auf unsere Umwelt hatten.

War diese Entwicklung - provokant formuliert - nun eine 80 bis 90 Jahre dauernde wissenschaftliche und politische „Fahrlässigkeit“? Oder war es vielmehr ein „noch nicht die Zusammenhänge erkennen und verstehen können“, ein „,noch nicht nachweisen und messen können“? Generell also die menschliche Unzulänglichkeit in die „Zukunft blicken zu können“, sprich die langfristigen Umweltauswirkungen unseres Tuns und unserer neuen technischen Errungenschaften verlässlich einschätzen zu können. 
Ich denke, dieses Beispiel sollte aufzeigen und uns lehren, wie wichtig es daher ist, zukünftige Auswirkungen von neuen Technologien möglichst weitreichend, systematisch und verlässlich abzuschätzen. Den Überlegungen zur Technikfolgenabschätzung der Entwicklung von Nanotechnologie widmet sich daher auch der erste Beitrag in diesem Themenheft.

Der Nachweis und das Monitoring dieser neuartigen synthetisierten Nanomaterialien in unseren Umweltmedien und nicht zuletzt deren Sicherheitsbewertung werden dadurch erschwert, dass nicht nur die chemische Spezies und die Menge bzw. Dosis entscheidend sind, sondern auch andere physikalisch-chemische Parameter, wie Partikelform, Struktur, spezifische Oberflächeneigenschaften, Größe und Verteilung eine wichtige Rolle spielen. Die Herausforderungen, die hierbei im Umweltmonitoring und beim Nachvollziehen der wirksamen Mechanismen auf uns zukommen, sind vielschichtig. Mit einigen Überlegungen und Lösungsansätzen dazu beschäftigt sich ein weiterer Artikel in dieser Ausgabe.

Obwohl von Jahr zu Jahr mehr Nanokonsumprodukte auf den österreichischen und v. a. europäischen Markt drängen, gibt es kaum belastbare und umfassende Informationen, in welchen Produkten welche Nanomaterialien hinsichtlich Zusammensetzung, Form und Menge enthalten sind. Genaue Produktkennzeichnungen fehlen größtenteils und damit eine wesentliche Möglichkeit, das Potenzial entsprechend zu quantifizieren. Bestenfalls finden sich grobe Hochrechnungen und Abschätzungen für einzelne Materialien. Daher kann sich zurzeit sowohl die Abwasser- wie auch die Abfallwirtschaft nur schwierig auf nanohaltige Abwässer bzw. Abfallströme einstellen. Erste Einblicke und Erkenntnisse dazu werden in drei weiteren fachspezifischen Beiträgen dargestellt.

Ob und in welcher Weise die Zunahme von nanomaterialhaltigen Produkten für die Abfall- und Abwasserwirtschaft von entsprechender Relevanz sein wird, wird v. a. die Zukunft zeigen. Jedenfalls heißt es aufmerksam zu bleiben, dort, wo der Mensch beginnt, synthetisierte und derzeit noch schwer nachweisbare Stoffe zunehmend zu nutzen und in vielfältiger Weise in der Umwelt zu verteilen. Stoffe nämlich, welche die Natur per se so nicht kennt und auf die die Umwelt daher auch nicht mit effektiven natürlichen Schutz- und Abbaumechanismen vorbereitet ist.

\author{
Univ.-Prof. DI Dr. M. Huber- \\ Humer $(\square)$ \\ Department für Wasser, Atmosphäre \\ und Umwelt, Institut für Abfallwirtschaft, \\ Universität für Bodenkultur Wien, \\ Muthgasse 107, \\ 1190 Wien, Österreich \\ marion.huber-humer@boku.ac.at
}

